\title{
The Implementation, Flexibility and Reasonable Selection of Bonus-Point Policy for Minority Candidates in College Entrance Examination from the Perspective of Educational Equality
}

\author{
Jianming $\mathrm{Du}^{1, *}$ \\ ${ }^{1}$ Law School, Inner Mongolia University, Hohhot, Inner Mongolia, China \\ *Corresponding author. Email: djm207@126.com
}

\begin{abstract}
Preferential policy that awards bonus points in national college entrance examination for ethnic students is nothing new. Thanks to such preferential means as bonus point or score deduction, the policy favors ethnic minorities as for the distribution of educational resources, thus endowing them with equal access to educational opportunities. However, the specific implementation of such policy varies. Among them, the execution plan based on specific "identity" and "culture" has inadvertently spawned issues such as "reverse discrimination" and "ethnic inequality". The research results reveal that bonus-point policy based on "region" serves as the best to maximize benefits and promote ethnic equality and unity.
\end{abstract}

Keywords: Educational equality, Ethnic education, Substantive Equality, Region.

\section{INTRODUCTION}

A series of preferential education policies for ethnic minorities are employed in China to better the development of education in ethnic minority areas. Bonus-point policy for minority candidates in college entrance examination that favors ethnic students covers "lenient admission, preferential admission, appropriate score reduction, appropriate awarded marks". In this way, the inferior position of ethnic minorities in the starting point of education has been made up, safeguarding their right to education. Combined with the specific implementation of bonus-point policy in various provinces and cities published online in 2015, the author divides them into four camps based on main implementation basis and standards. The first is "identity", that is, the special identification from local education administrative department is required for ethnic examinees to enjoy the policy. In reality, "priority admission without bonus points" applies to all minority examinee of Shanxi

\footnotetext{
*Fund: This paper is the periodic result of the project "Research on the Development Path of Ethnic Minority Education Equality" (2017NDB076) of Inner Mongolia Social Science Planning Office.
}

Province, "bonus points for minority examinee" is adopted by 7 provinces and cities including Beijing, while the rest provinces employ "differential treatment, special identification", namely, examinees of some ethnic groups enjoy bonus points, while that of other ethnic groups enjoy "priority admission". The second is "region", that is, areas inhabited by ethnic minorities are taken as criteria. Minority examinees in concentrated area of nationalities or specific areas enjoy bonus points, while those living in areas inhabited both by ethnic minorities and the Han people enjoy extra points as appropriate or priority admission. However, much leeway is allowed in implementation. For example, only minority examinees of the She, Hui and Gaoshan in concentrated area of nationalities enjoy bonus point in Fujian, while both minority and Han examinees in concentrated area of nationalities are entitled to varied bonus point in Hunan and Guangdong. The third is "culture". Native language is highlighted and taken as the main basis for implementation, that is, "whether the test paper is written in the native language or not is considered as the standard". Except for Jilin Province, Heilongjiang allows a double standard of bonus points for designated minority examinees within its 
jurisdiction. The fourth refers to other categories launched in such areas as Tibet, Qinghai and Xinjiang. To be frank, it is impossible to distinguish the implementation of this policy by any definite criteria in China's provinces or regions. However, the implementation following various standards in regions leads to the disparity and inequality of educational opportunities and resources for students sharing ethnic identity while living in different regions. This further indicates that policies based on substantive equality will be consumed due to chaotic implementation and differences, which may even beget new issues and contradictions.

\section{RATIONALITY OF THE FLEXIBILITY OF BONUS-POINT POLICY FOR MINORITY CANDIDATES IN COLLEGE ENTRANCE EXAMINATION}

\subsection{Confusing "Differential Treatment"}

Regardless of implementation standard and basis they follow, "differential treatment" is commonly employed as logical premise by specific implementations of bonus-point policy. In other words, all examinees divided based on their nationality, either Han or minority, are endowed with diverse preferential treatment and care. Such distinction can be performed based on "national identity". Back in the 1950s, China followed the practice of the Soviet Union in launching an ethnic identification campaign. More than 400 ethnic groups applied then, and 56 were finally identified. Both history and reality prove the huge gap between the ethnic minorities and the Han in economic development and social progress. Given the long-term vulnerable position of ethnic minorities and ethnic minority areas in economic and social progress, preferential treatment, assistance and care have been deployed to ethnic minorities in such aspects as school admission, enrollment and employment. Such "differential treatment", though divorcing from formalistic rule of law that "all people are equal before the law", objectively contributes to the substantive equality of the right to education of ethnic minorities.

Plato takes that "indiscriminate equality of all equals inequality." [1] Even if ethnic minorities do suffer from inequality in educational opportunities, the tilt in the supply of educational products and the distribution of educational opportunities concern the fairness of social system, so such tilt must be justified. Currently, theory of justice proposed by American thinker Rawls has been widely taken as the theoretical basis of preferential policies for ethnic education by Chinese scholars, thus enhancing its legitimacy. Rawls's theory of justice demands that "all parts of society and everyone with opposing motives and endowments should enjoy roughly equal prospects for education and achievement. The will of those who boast same abilities and aspirations should not be influenced by their social origin." [2] Therefore, the principle of justice in Rawls' theory requires that "those with less talent and from less advantaged social positions deserve more social attention in order to treat all people equally and to provide truly equal opportunities. The idea is to compensate for accidental skewing on the basis of equality." [3] To eliminate and overcome the inferiority in education caused by economic backwardness, conservative ideas and unbalanced allocation of educational resources, bonus points are given to minority candidates in college entrance examination and enrollment, and preferential treatment is given to them in different links of education, thus ensuring "equal access to equal opportunities" (Rawls) and substantive equality. As a result, bonus-point policy for minority candidates in college entrance examination and numerous preferential policies for ethnic education conform to the insight of Rawls: "to maximize the interests of the most disadvantaged lives up to the principles of equity and justice." [4]

Rawls's theory of justice does prove the legitimacy of bonus-point policy for minority candidates in college entrance examination. However, the institutional arrangement made by the theory to the social macro structure lies in value guidance for the allocation of educational resources. That is to say, it can only guarantee its purposefulness. The legitimacy of any public policy involves both ends and means. The first can only ensure correct direction, while the latter represents correctness of method selection, the key to ensure the smooth realization of results. The wrong approach not only makes the goal difficult to achieve, even counterproductive. The "differential treatment" and the following "reverse discrimination" need to be solved urgently. Han and minority examinees living in concentrated area of nationalities and basically sharing educational resources and cultural background are entitled with varied educational opportunities due to "differential treatment", which naturally leads to "reverse discrimination". However, "differential treatment" 
has been implemented in most provinces and cities in China. At present, only provinces including Hunan and Guangdong allow bonus points for Han students in concentrated area of nationalities. For example, Hunan Province stipulates that "the admission threshold for minority candidates from prefectures, counties and townships of areas inhabited by ethnic minorities can be lowered by 20 points, while that for Han students from such areas can be lowered by 10 points." The practice of Guangdong is more reasonable: "as for juror college examination, 10 points will be added to the total score in the unified examination of general knowledge course for minority candidates and Han students in areas inhabited by ethnic minorities." The bonus points adopted by most provinces and cities in China has increased the enrollment rate of ethnic minority examinees in the college entrance examination and promoted substantive equality. However, such simplistic thinking in dealing with ethnic relations exaggerates "reverse discrimination", which greatly tarnishes the value connotation of equality while realizing substantive equality.

\subsection{Worry Behind Identity Designation}

The access to bonus-point policy for minority candidates in college entrance examination requires ethnic identity, which is special attention to the minority by modern state under the rule of law based on the consideration of the history and reality, economy and society, survival and development of the ethnic minorities. All ethnic minorities within the administrative region enjoy equal access to the policy, which meets the theoretical requirement of respect for human rights and justice of rights, as well as the practical need of ethnic equality and ethnic unity. An overview of implementation in province and city reveals that implementation programs adopted by many regions have deviated from the rule of law requirement of equal rights. In Heilongjiang Province, for example, it's stipulated that colleges and universities in the province shall add 10 points to the total score of students from Oroqen, Hezhe, Ewenki, Kirgiz, Mongolia, Xibe and Russian ethnic in the unified examination. Similar practice can be observed in Inner Mongolia. Mongolian, Dawur, Oroqen, Ewenki and Russian students can be admitted 10 points lower. The designation of the aforesaid ethnic minority by the competent educational administrative departments within their respective administrative areas shall be regarded as a premise for policy bonus based on identity. However, it's worth attention to reflect on the justification for such move. According to the sixth population census, the population of Inner Mongolia Autonomous Region in 2010 consists of permanent residents of 55 of the 56 ethnic groups, except the Lhoba nationality. Among them, 19.215 million are Han, accounting for 77.8 percent of the total population, and 5.4914 million are ethnic minorities. Among the ethnic minorities, the Mongolian population is 4.411 million, accounting for 80.3 percent of the ethnic minority population. [5] Given the geographical distribution of China's college enrollment index, coupled with the influence of bonus-point policy, if examinees with the national identity of "the three ethnic minorities": Dawur, Oroqen, and Ewenki, are allowed to bonus points, the progressive logic of the guarantee of "minority rights" among minorities is satisfied. But how is it reasonable to give special care to the Mongols, the most populous ethnic group after the Han ethnic group. Is it justified to treat other ethnic groups differently than the five mentioned above?

In addition, Ningxia's bonus-point policy stipulates that "the total score of general knowledge courses in the college entrance examination for students of other ethnic minorities except Hui nationality shall be increased by 10 points, while that for students of Hui nationality shall be increased by 20 points." Preferential policy that awards bonus points in national college entrance examination for ethnic students and other preferential policies for ethnic minority have always been formulated to eradicate the rigidity of formal equality, thus achieving substantive equality. However, such implementation method undoubtedly begets new inequality on the road leading to substantial equality, which naturally leads to ethnic discrimination among ethnic minorities and aggravates ethnic estrangement. What's more, Xinjiang stipulates that examinees from 11 ethnic minorities, such as Uygur and Kazak, enjoy bonus points, and those whose parents are both from the above-mentioned ethnic groups will get 50 points more, while those whose parents are from the above-mentioned ethnic groups will get 10 more points. As is known to all, inter-ethnic marriage implies the deep communication and integration of different ethnic cultural backgrounds, as well as affects the social harmony and stability within the nation. In his book Assimilation of American Life, Milton Gordon, a famous American sociologist, proposes seven variables to study and evaluate ethnic integration, among which interracial 
marriage is taken as the most important aspect. However, Xinjiang's practice has virtually hindered inter-ethnic marriage, and the vulnerable position of children of intermarriage in college entrance examination will inevitably stymie in-depth ethnic integration.

\subsection{The Rationality of "Cultural" Guarantee}

As for the implementation of bonus-point policy for minority candidates in college entrance examination, whether the test paper is written in the native language or not is considered as the standard to bonus points, which applies in Jilin and Heilongjiang Provinces. Jilin Province stipulates that ethnic minority examinees who do not use their own written language will get 5 points added to their actual scores, while those who do will get 10 points added. Since the spoken and written languages of ethnic minorities serve as an integral of their cultures, the protection of such languages is essential for the protection of the rights of ethnic minorities in China. Both the Constitution and Law of the People's Republic of China on Regional National Autonomy stipulate in principle the protection of the spoken and written languages of ethnic minorities, stating that "all ethnic groups have the freedom to use and develop their own spoken and written languages". Protection of the spoken and written languages of ethnic minorities can also be observed in Education Law in 1995, Decision on Deepening Reform and Accelerating the Development of Ethnic Education in 2002, and Regulations on the Implementation of the Law of the People's Republic of China on Regional National Autonomy in 2005. In view of the importance of the national culture represented by the spoken and written languages of ethnic minorities, the Chinese government has employed various measures to protect the rights of the spoken and written languages of ethnic minorities. For example, China's Election Law and Criminal Procedure Law of the People's Republic of China respect and guarantee the right of ethnic minorities to use their own spoken and written languages in political life and judicial proceedings. Therefore, granting bonus points to ethnic students who answer in their own written languages has become a legal guarantee of their rights and interests. The essence of these plausible rights thinking is the simplification of rights. Protection of the rights to use ethnic minority languages in Election Law and Criminal Procedure Law of the People's Republic of China is based on the instrumental value of language in realizing ethnic minority political rights and litigation rights, which cannot be realized without the necessary means of language. However, the bonus points to ethnic examinees who use ethnic minority language may run counter to the value orientation of this policy.

It's widely known that bonus-point policy for minority candidates in college entrance examination, like other ethnic education policies, aims to improve the equality of opportunity in the education of ethnic minorities and realize the substantive equality of education. Minority examinees shall be compensated by means of bonus points, score reduction or priority admission to achieve such goals. Poor cultural quality caused by backward economy, inconvenient transportation, conservative ideas and unbalanced distribution of educational resources in ethnic minorities and minority areas explains such compensation. However, the causal relationship between such poor cultural quality of ethnic students caused by social structural factors and the learning and use of their own language and characters has not been proved, so it is not valid to take this standard as the basis of bonus-point policies. Further study raises the question: if the study of their own national language and writing will lead to poor cultural quality, thus requiring bonus points, then does such statement apply to candidates learning English, Russian, or Japanese? It needs to be pointed out that the author can fully understand the cultural value of minority languages, as well as the significance and value of diversified national cultures to minority nationalities themselves and all human beings. The author takes that the use of ethnic language as the premise to bonus points lacks legitimacy, as the logic behind is the causal relation between national language learning and poor quality of ethnic students, which results in national estrangement, or even ethnic discrimination. In this way, the continuation and inheritance of ethnic culture by minority students will be hamstringed. The guarantee of minority languages in education can also be realized by strengthening bilingual education or other ways such as scholarship and aid. 


\section{DIRECTION OF ADJUSTMENT OF BONUS-POINT POLICY FOR MINORITY CANDIDATES IN COLLEGE ENTRANCE EXAMINATION}

The practice of taking "region" as criteria for preferential policy that awards bonus points in national college entrance examination for ethnic students originates from the theoretical interpretation of unbalanced education in China. The representative of the new institutional economics, the famous Swedish economist Gunnar Muirdal cannot be omitted. As the winner of the Nobel Prize in Economics, he takes the social economy as a whole, analyzes the causality of various relevant factors from the perspective of social dynamics, thus proposing the "circulatory accumulation theory". The theory holds that various related factors in the dynamic process of social and economic development are related to each other, influence each other and serve as cause and effect to each other. Any two of the elements of dynamic link enjoy causal relationship, one of which defines direction, and its change will cause the change of the other element, while the other will in turn incur intensified development. In view of the nonequilibrium among social and economic elements, such circular movement is not a simple circular circulation, but a circular movement with cumulative effect, which constitutes "circulatory accumulation theory". Muirdal's An American Dilemma: The Negro Problem and Modern Democracy published in 1944 elucidated the theory through examples of poverty and race relations: the discrimination and prejudice of white people lead to the poor material and cultural level of black people, while the economic poverty and lack of education of black people in turn enhance the discrimination of white people, which constitutes "discrimination breeds discrimination".

The theory contributes to the explanation and solution of the issue of unbalanced regional development in China. Muirdal proposed that "in the early stage of economic development, the government should prioritize areas with better conditions for better investment efficiency and faster economic growth, thus driving the growth of other areas through diffusion effect. However, when the economy develops to a certain stage, the infinitely expanding gap between the rich and the poor caused by circulatory accumulation should be avoided. The government must make a series of special policies to stimulate the development of backward areas, so as to narrow the economic disparity." [6] Given the vicious circle between unbalanced development of China's education, poverty and educational backwardness in the minority areas, and poor cultural quality of the minorities, active state intervention including system arrangement and policy care should be conducted to help backward minority areas get rid of predicament. The implementation of bonus-point policy for minority candidates in college entrance examination, a national policy in the favor of ethnic education, should take exclusively "region" as standard, promote the quantity and quality of human resources in national regions through positive policy intervention, and promote the regional development of ethnic education, thus substantiating the benign interaction and harmonious development between education resource allocation and economy progress in minority regions.

First, the practice standard of "region" as for bonus-point policy for minority candidates in college entrance examination helps to curb the formation of "reverse discrimination". In reality, all countries will adopt preferential policies for ethnic minorities in response to ethnic relations, which contributes to the protection of rights and interests of ethnic minorities, reducing ethnic conflicts and stabilizing ethnic relations. However, improper implementation of such policies will damage the interests of the ethnic group involved, which leads to sour ethnic relations. The examinees from Han and minority nationalities living in the same time and space share life experience, which constructs the inter-embedded community structure, making them share educational resources and suffer consequences of unbalanced education. However, differences in "identity" widen their distance and give rise to barriers. The policy implementation based on "region" fully considers the fact that China's ethnic integration and ethnic exchanges are constantly close, which guarantees the vital interests of minority students, and eliminates the prejudice of Han students. The dialectical unity of formal equality and substantive equality of policy orientation within the region maximize the effect and benefit.

Second, the practice standard of "region" as for bonus-point policy for minority candidates in college entrance examination helps to exterminate another form of "ethnic inequality". In addition to the inequality between the Han and the minority nationalities, ethnic inequality also refers to the inequality between minority nationalities. The 
implementation of bonus-point policy for minority candidates in college entrance examination strives to achieve substantive equality of educational opportunities through preferential treatment, care and assistance. However, the mixed and superficial implementation in provinces and cities lead to varied preferential treatment for ethnic minorities from different ethnic groups, which weakens the policy effect, and gives rise to another form of "ethnic inequality". The move to take "region" as standard for bonus-point policy for minority candidates in college entrance examination may eliminate ethnic estrangement and create a favorable policy environment for ethnic equality.

Lastly, the practice standard of "region" as for bonus-point policy for minority candidates in college entrance examination is in accord with the trend of ethnic education, contributing to eliminating ethnic differences, realizing ethnic equality, ethnic unity and common prosperity of all ethnic groups. The Chinese government has always attached great importance to ethnic education. Released in 2015 by the State Council, Decision of the State Council on Expediting the Development of Ethnic Education points out that the policy of combining ethnic factors with regional factors should be followed, the differentiated regional policies should be perfected, and district planning and targeted guidance should be ensured to cement the foundation for development, narrow the growth gap, and promote educational equity. No ethnic minority or region should be left behind, so as to promote the all-round development of ethnic education. The year 2016 witnessed the release of Guidance on Accelerating the Development of Education in Central and Western China by the State Council. Accelerating the development of education for ethnic minorities is taken as one of the priorities. Special support should be taken to favor ethnic minorities and ethnic minority areas in various policies. What's more, the popularization and quality of education at all levels and of all types should be rapidly improved to realize leapfrog development of education in ethnic minority areas. All these reveal that the Chinese government's focus on ethnic education can be explained by her ambition to realize ethnic equality with emphasis on regional progress and through eliminating the consequences of unbalanced development of educational resources.

\section{CONCLUSION}

Against the macro background of unbalanced education development in the period of social transformation in China, the "identity" standard in the college entrance examination of ethnic minorities is changed to the "regional" standard. In this way, the unbalanced state in ethnic regions is faced squarely and preferential policies of different strength are adopted, and the examinees of all ethnic groups in the same area are treated equally. It not only guarantees the vital interests of minority examinees, but also eliminates the inner estrangement of Han examinees. The "differential treatment" between regions is adopted to achieve substantial equality, and take into account the equality of form within the region, the dialectical unity of which will jointly promote the equal realization of the right to education of Chinese citizens.

\section{AUTHORS' CONTRIBUTIONS}

This paper is independently completed by Jianming Du.

\section{REFERENCES}

[1] [Ancient Greece] Plato. The Law [M]. Translated by Zhang Zhiren, He Qinhua, Shanghai: Shanghai People's Publishing House. 2001, p.168. (in Chinese)

[2] He Huaihong. Justice as Fairness: Explanation of Rawls' On Justice [M]. Ji'nan: Shandong People's Publishing House. 2002, p. 57. (in Chinese)

[3] [America] Rawls. Justice as Fairness [M]. Translated by Yao Dazhi, Shanghai: SDX Joint Publishing Company. 2002, p. 102. (in Chinese)

[4] [America] Rawls. A Theory of Justic [M]. Translated by He Huaihong and others, Beijing: China Social Sciences Press. 1988, p. 60. (in Chinese)

[5] Yang Yuan. The population development status of ethnic minorities in Inner Mongolia [J]. Inner Mongolia Statistics, 2015, (5). (in Chinese)

[6] Baidu Encyclopedia: Economic Radiation. http://baike.haosou.com/doc/78892458163340.html.2016-7-24. (in Chinese) 